\title{
Endoscopic finding and treatment outcome of children with Helicobacter pylori infection in lower northern Thailand
}

\author{
Chayakamon Niyasom, Thitima Ngoenmak*, Suwannee Uthaisangsook
}

\begin{abstract}
Background: Helicobacter pylori infection is one of the predisposing factors for gastritis, peptic ulcer, and duodenal ulcer. Definite diagnosis of $H$. pylori infection is important in planning effective medical management. However, confirming the diagnosis through bacterial culture takes a number of days, and thus delays treatment.

Objectives: To examine endoscopic findings in children associated with chronic abdominal pain and $H$. pylori infection to aid in early diagnosis. We also evaluated treatment outcome of $H$. pylori infection.

Methods: A retrospective study was performed by reviewing the medical records of children under 15 years of age with chronic abdominal pain who underwent esophagogastroduodenoscopy (EGD) between 2011 and 2017. According to 2016 Joint ESPGHAN/NASPGHAN Guidelines, H. pylori infection was defined by positive tests for both histopathology test and rapid urease test (RUT). The EGD finding, RUT, histopathologic finding, and treatment outcome were recorded.

Results: Forty-eight children presented with chronic abdominal pain (male $47.9 \%$, female $52.1 \%$, mean age was $8.44 \pm 2.97$ years). Twelve children out of 48 had $H$. pylori infection (12/48, 25\%). Eight among the 12 children had antral nodularity (8/12), wherein there was no antral nodularity in children without $H$. pylori infection $(0 / 36)$. This difference was statistically significant $(P<0.001)$. Sensitivity and specificity of antral nodularity finding for $H$. pylori infection were $66.7 \%$ and $100 \%$, respectively. Eradication of $H$. pylori infection with standard regimen improved the abdominal pain within 4 weeks.

Conclusion: The occurrence of antral nodularity in endoscopic finding was significantly associated with $H$. pylori infection. In addition, antral nodularity finding showed a good sensitivity and high specificity for the diagnosis of H. pylori infection.
\end{abstract}

Keywords: antral nodularity; chronic abdominal pain; esophagogastroduodenoscopy; Helicobacter pylori; rapid urease test

Helicobacter pylori infection acquired in early childhood increases the risk of $H$. pylori-related complications later in life, such as gastritis, peptic ulcer, duodenal ulcer, and chronic abdominal pain. The prevalence of $H$. pylori infection is higher in developing countries than that in developed countries. In
Thailand, the prevalence of $H$. pylori infection is $15 \%-20 \%$ that increases with age [1].

Diagnostic procedures are composed of the tissue biopsies from esophagogastroduodenoscopy (EGD) for the rapid urease test (RUT) and histopathology test. One of the abnormal

*Correspondence to: Thitima Ngoenmak, Department of Pediatrics, Faculty of Medicine, Naresuan University, Phitsanulok 65000, Thailand, e-mail:Thitiman@nu.ac.th

Department of Pediatrics, Faculty of Medicine, Naresuan University, Phitsanulok 65000, Thailand

¿ Open Access. ๑ 2019 Chayakamon Niyasom et al., published by Sciendo. (c) BY-NC-ND This work is licensed under the Creative Commons Attribution NonCommercial-NoDerivatives 4.0 License. 
endoscopic findings is antral nodularity, which defines as a cobblestone-like mucosa with nodules varying in size from 2 to $4 \mathrm{~mm}$ and are covered with smooth, non-ulcerated mucosa. A previous study [2] showed that the percentage of antral nodularity, erythematous mucosa, and normal endoscopic findings in $H$. pylori infection children are 47.4, 24.8, and 22.2 , respectively.

H. pylori infection status is defined as positive RUT and histopathology test of gastric biopsy. Sensitivity and specificity of the RUT test were $75 \%-100 \%$ and $99-100 \%$, respectively, and of the histopathology test were $66 \%-100 \%$ and $100 \%$, respectively $[3,4]$. Gold standard to diagnose $H$. pylori infection is bacterial culture, which is time consuming. Therefore, other diagnostic tools are being explored.

The purpose of this study was to examine endoscopic findings in children associated with chronic abdominal pain and H. pylori infection to aid in early diagnosis. We also evaluated treatment outcome of $H$. pylori infection.

\section{Methods}

The study was conducted in the Gastroenterology Unit, Department of Pediatric, Naresuan University Hospital, located in lower northern Thailand. This study was approved by the Institutional Review Board (IRB) of Naresuan University (certificate of approval no. 0225/61). We retrospectively reviewed medical records of 48 children below the age of 15 years with chronic abdominal pain who underwent EGD between 2011 and 2017. Each child underwent six gastric biopsies, two from the antrum and the body of stomach for RUT and four from esophagus, antrum, body, and duodenum for histopathology. No antibiotic and proton pump inhibitor (PPI) were taken within 4 weeks and 2 weeks, respectively, before the procedure. The abnormality of endoscopic finding was also recorded such as antral nodularity and erythematous mucosa. All infected children were treated with triple therapy using 14 days of amoxicillin $(50 \mathrm{mg} / \mathrm{kg} /$ day $)$ and clarithromycin $(20 \mathrm{mg} / \mathrm{kg} /$ day $)$, plus 30 days of omeprazole (2 mg/kg/day) [3].

\section{Identification of $\boldsymbol{H}$. pylori infection status}

The positive RUT and histopathology test of gastric biopsy results determined the $H$. pylori infection status of the subjects. The gastroenterologist who performed the endoscopy interpreted the RUT at 1 hour and 24 hours. The color change from yellow to pink indicated positive RUT. Gastric biopsy specimens were stained with hematoxylin and eosin to evaluate the severity of gastritis in reference of the updated Sydney histological scoring system. The same histopathologist also stained the specimens with Giemsa to detect H. pylori infection.

\section{Statistical methods}

Demographic data, EGD finding, histopathology, and the correlation with $H$. pylori infection were analyzed using Pearson's chi-square test and Fisher's exact test. The $P$ values of less than 0.05 were considered as statistically significant.

\section{Result}

Among 48 children with chronic abdominal pain, there were 23 males (47.9\%) and 25 females (52.1\%), whose mean age was $8.44 \pm 2.97$ years. The most common age range was 6-10 years $(62.5 \%)$. Twelve of 48 children were diagnosed with $H$. pylori infection $(12 / 48,25 \%)$. There were five males and seven females who had $H$. pylori infection with the mean age of $9.78 \pm 2.16$ years (Table $\mathbf{1}$ ).

Eight of 12 children were found to have antral nodularity $(8 / 12)$, while there was no antral nodularity found in children without $H$. pylori infection (0/36). This difference was statistically significant (Table 2). In this study, sensitivity and specificity of antral nodularity finding for $H$. pylori infection were $66.7 \%$ and $100.0 \%$, respectively.

Ten of 12 children who had H. pylori infection had normal appearance of esophagus and duodenum. However, 11 children with $H$. pylori infection had erythematous mucosa

Table 1. Demographic data of children with chronic abdominal pain and Helicobacter pylori infection

\begin{tabular}{lcc}
\hline Demographic data & $\begin{array}{c}\text { Chronic abdominal } \\
\text { pain, } \mathbf{n}(\%)\end{array}$ & $\begin{array}{c}\boldsymbol{H} \text {. pylori infection, } \\
\mathbf{n}(\%)\end{array}$ \\
\hline Sex & $23(47.9)$ & $5(41.7)$ \\
Male & $25(52.1)$ & $7(58.3)$ \\
Female & $48(100.0)$ & $12(100.0)$ \\
Total & & \\
Age (years) & $8(16.7)$ & $0(0.0)$ \\
$0-5$ & $30(62.5)$ & $8(66.7)$ \\
6-10 & $10(20.8)$ & $4(33.3)$ \\
$11-15$ & $48(100.0)$ & $12(100.0)$ \\
Total & $(1.0-14.0)$ & $(6.1-14.0)$ \\
Age range (years) & & \\
\hline
\end{tabular}


Table 2. Presence of antral nodularity in children with Helicobacter pylori infection

\begin{tabular}{lccc}
\hline Antral nodularity & \multicolumn{2}{c}{. pylori infection, $\mathbf{n}$} & \multirow{P}{*}{} \\
\cline { 2 - 3 } & Positive & Negative & \\
\hline Positive & 8 & 0 & $<0.001$ \\
Negative & 4 & 36 & \\
Total & 12 & 36 & \\
\hline
\end{tabular}

in their stomach. Among these 11 children with erythematous mucosa of stomach, 5 children had positive RUT at 1 hour and 6 children had positive RUT at 24 hours, respectively. Positive RUT at 1 hour and 24 hours was not significantly correlated with gastric erythematous mucosa $(P=0.377)$. In addition, the presence of antral nodularity was not significantly related to the presence of gastric erythematous mucosa $(P=0.14)$. Triple therapy alleviated the abdominal pain in all children with $H$. pylori infection within 4 weeks, $8.3 \%$ and $58.3 \%$ of which improved within 1 week and 2 weeks, respectively.

\section{Discussion}

H. pylori infection acquired in childhood years is one of the most common causes of chronic abdominal pain [5, 6]. In this study, the prevalence of $H$. pylori infection in chronic abdominal pain was $25 \%$, which was higher than that in the previous study. This is likely because patients could access to pediatric gastroenterologist directly. This study found that the mean age of $H$. pylori-infected children was $9.78 \pm 2.16$ years, which is comparable to the study of Motamed et al. [2], showing the mean age of $7.91 \pm 3.93$ years among 3,031 children respondents.

The gold standard test for the diagnosis of $H$. pylori infection is a bacterial culture that takes 1 month to procure the result; therefore, other diagnostic tools are important to aid in early diagnosis and treatment. In this study, antral nodularity in endoscopic finding was significantly associated with H. pylori infection $(P<0.001)$ but not with gastric erythematous mucosa. This finding is congruent with the retrospective study conducted by Luzza et al. [5] on 174 subjects showing antral nodularity associated with $H$. pylori infection. Several studies have reported sensitivity and specificity of antral nodularity for the diagnosis of $H$. pylori infection of $40 \%-91.6 \%$ and $85.7 \%-100 \%$, respectively $[5,7,8]$, which are comparable to our study showing sensitivity and specificity of $66.7 \%$ and $100.0 \%$, respectively. Our study did not demonstrate the correlation between antral nodularity and gastric mucosal erythema.
Regarding $H$. pylori eradication, a retrospective study [9] conducted in 2004 showed that $70 \%$ of the 149 children treated with triple regimens (PPI with amoxicillin and clarithromycin) improved within 1 week and $81 \%$ of the same respondents improved within 2 weeks. This finding, however, is incomparable to our study, which revealed a slow improvement of $8.3 \%$ within 1 week and $58.3 \%$ within 2 weeks.

Our study was limited because of small sample size of children; therefore, we did not demonstrate the correlation between antral nodularity and gastric mucosal erythema. Moreover, the reason for slower improvement of triple therapy in H. pylori-infected children is not clear; somehow, the further study of antibiotic susceptibility testing would be beneficial to improve management.

\section{Conclusion}

The antral nodularity in endoscopic finding is significantly associated with $H$. pylori infection. In addition, the presence of antral nodularity is a good predictor of $H$. pylori infection.

Author contributions. $\mathrm{CN}$ and TN contributed substantially to the conception and design of this study. All authors contributed substantially to the acquisition and interpretation of the data. TN drafted the manuscript. All the authors contributed substantially to its critical revision, approved the final version submitted for publication, and take responsibility for the statements made in the published article.

Acknowledgment. The study was supported by the research funding of Naresuan University. The authors gratefully acknowledge thanks to Miss Kornthip Jeephet, Statistics Technical Officer, Research Center, Faculty of medicine, Naresuan University, for statistical analysis. The authors also thank the operative nurses of the Naresuan University Hospital for collecting patients' history.

Conflict of interest statement. The authors have completed and submitted the International Committee of Medical Journal Editors Uniform Disclosure Form for Potential Conflicts of Interest. None of the authors disclose any conflict of interest.

\section{References}

[1] Perez-Perez GI, Taylor DN, Bodhidatta L, Wongsrichanalai J, Baze WB, Dunn BE, et al. Seroprevalence of Helicobacter pylori infections in Thailand. J Infect Dis. 1990; 161:1237-41. 
[2] Motamed F, Doroudian R, Najafi M, Monajemzade M, Marashi SM, Arastoo L, et al. Helicobacter pylori infection: clinical, endoscopic and pathological findings in Iranian children. Int J Pediatr. 2014; 2:9-17.

[3] Jones NL, Koletzko S, Goodman K, Bontems P, Cadranel S, Casswall $\mathrm{T}$, et al. Joint ESPGHAN/NASPGHAN guidelines for the management of Helicobacter pylori in children and adolescents (Update 2016). J Pediatr Gastroenterol Nutr. 2017; 64:991-1003.

[4] Roma-Giannikou E, Roubani A, Sgouras DN, Panayiotiu J, Van-Vliet C, Polyzos A, et al. Endoscopic tests for the diagnosis of Helicobacter pylori infection in children: validation of rapid urease test. Helicobacter. 2010; 15:227-32.

[5] Luzza F, Pensabene L, Imeneo M, Mancuso M, Contaldo A, Giancotti L, et al. Antral nodularity identifies children infected with Helicobacter pylori with higher grades of gastric inflammation. Gastrointest Endosc. 2001; 53:60-4.

[6] Kaewplang P. Prevalence and clinical manifestation of Helicobacter pylori infection in pediatrics at Surin Hospital. Thai J Pediatr. 2012; 55:132-9.

[7] Mazigh Mrad S, Abidi K, Brini I, Boukthir S, Sammoud A. Nodular gastritis: an endoscopic indicator of Helicobacter pylori infection in children. Tunis Med. 2012; 90:789-92.

[8] Łazowska-Przeorek I, Kotowska M, Banasiuk M, KarolewskaBochenek K, Banaszkiewicz A, Gawrońska A, et al. Value of antral nodularity for the diagnosis of Helicobacter pylori infection in children. Med Sci Monit. 2015; 21:1827-30.

[9] Kato S, Konno M, Maisawa S-I, Tajiri H, Yoshimura N, Shimizu T, et al. Results of triple eradication therapy in Japanese children: a retrospective multicenter study. J Gastroenterol. 2004; 39:838-43. 